\title{
Matching with Stochastic Arrival
}

\author{
[Extended Abstract] ${ }^{*}$ \\ Neil Thakral \\ Harvard University \\ 1805 Cambridge St. \\ Cambridge, MA \\ nthakral@fas.harvard.edu
}

\begin{abstract}
We study matching in a dynamic setting, with applications to the allocation of public housing. In our model, objects of different types that arrive stochastically over time must be allocated to agents in a queue. For the case that the objects share a common priority ordering over agents, we introduce a strategy-proof mechanism that satisfies certain fairness and efficiency properties. More generally, we show that the mechanism continues to satisfy these properties if and only if the priority relations satisfy an acyclicity condition. We then turn to an application of the framework by evaluating the procedures that are currently being used to allocate public housing. The estimated welfare gains from adopting the new mechanism are substantial, exceeding $\$ 5,000$ per applicant.
\end{abstract}

\section{Categories and Subject Descriptors}

J.4 [Social and Behavioral Sciences]: Economics

\section{General Terms}

Economics

\section{Keywords}

Market design, dynamic matching, public housing allocation

\footnotetext{
*A full version of this paper is available at http://scholar.harvard.edu/files/nthakral/files/Thakral_match.pdf
} 